\title{
Two-dimensional electron gases in low-pressure metalorganic vapour phase epitaxially grown InGaP homojunctions
}

\author{
P.R. Hageman *, F.A.J.M. Driessen, G.J. Bauhuis, L.J. Giling \\ Department of Experimental Solid State Physics, RIM, Faculty of Science, University of Nijmegen, Toernooiveld, \\ 6525 ED Nijmegen, Netherlands
}

\begin{abstract}
We present modulation-doped ordered-InGaP/disordered-InGaP homojunctions grown, lattice matched to $\mathrm{GaAs}$, by metalorganic vapour phase epitaxy. Capacitance-voltage $(C-V)$ profiling techniques, temperature-dependent Hall, Shubnikov-De Haas and photoluminescence measurements have been used for characterization. The $C-V$ measurements show a narrow profile at the homointerface with an order of magnitude reduction in carrier density within $3 \mathrm{~nm}$. From temperature dependent Hall measurements, typical two-dimensional behaviour is observed with sheet carrier densities as high as $3.6 \times 10^{13} \mathrm{~cm}^{-2}(T<100 \mathrm{~K})$. No carrier freeze-out and constant mobilities around $850 \mathrm{~cm}^{2} \mathrm{~V}^{-1} \mathrm{~s}^{-1}$ below $T=100 \mathrm{~K}$ are observed. The $300 \mathrm{~K}$ channel conductivity of this new type of junction is $3.2 \times 10^{-3} \Omega^{-1}$, which is higher than reported in other two-dimensional electron gases. Shubnikov-De Haas measurements indicate the presence of two occupied excited subbands. The photoluminescence measurements clearly show a moving emission which involves the 2DEG.
\end{abstract}

\section{Introduction}

In opto-electronic devices, like solid-state lasers and solar cells, the use of the III/V semiconductor InGaP has certain advantages over the more often used AlGaAs. These benefits concern both physical properties and behaviour during MOVPE growth. In comparison with AlGaAs, the sensitivity of InGaP towards traces of humidity or oxygen during growth is neglegible. Probably, this causes the very low surface recombination rate of the InGaP/GaAs interface, which is as low as $1.5 \mathrm{~cm} \mathrm{~s}^{-1}$, about two orders of magni-

\footnotetext{
${ }^{*}$ Corresponding author.
}

tude lower than values found for the AlGaAs/ GaAs interface. Where $\mathrm{Al}_{x} \mathrm{Ga}_{1-x}$ As with $x>$ 0.22 suffers from the detrimental effect of carrier trapping by the so-called DX-centres, no such trapping occurs in InGaP at the lattice matched composition.

The MOVPE growth of InGaP has special consequences for its structural properties. Depending on the growth conditions, i.e. V/III ratio, growth temperature, growth rate and substrate orientation, the material is in a more or less ordered state. This ordering takes place on the group III sublattice and is of the CuPt-type, i.e. it consists of (GaP)(InP) superlattices in two $\langle 111\rangle$ directions. A consequence of this ordering is a decrease of the bandgap. For a completely 
ordered lattice, a decrease of $260 \mathrm{meV}$ was calculated as compared to the completely disordered alloy [1]. In practice, values between 100 and 190 $\mathrm{meV}$ are found. The existence of ordered domains influences optical and transport properties of the material. The optical emission properties are determined by spatially indirect recombination involving confined donors [2] and the transport properties are influenced by scattering at clusters of ordered material in the matrix of disordered material and, at low temperatures, by confined donor states [3].

By proper choice of the growth conditions during MOVPE of InGaP, one can make material with different bandgaps, but with the same composition (bandgap engineering). We used these special properties to grow for the first time, ordered/disordered InGaP homojunctions with a 2DEG at the interface. At low pressure, we deposited on a GaAs buffer a layer of highly ordered InGaP with a low bandgap. On top of this, an InGaP layer with high bandgap was grown (disordered material). Both InGaP phases were grown lattice matched to GaAs. This high gap layer consisted of an undoped spacer layer and a uniformly n-type doped layer, which causes the formation of the 2DEG at the ordered/disordered interface.

In this paper we present results on the optical and electrical characterization of this system.

\section{Experimental procedure}

The experiments were carried out in a horizontal, low pressure (20 mbar) MOVPE reactor. The ordered InGaP (o-InGaP, low $E_{\mathrm{g}}$ ) was grown with a V/III ratio of 125 and at $T=640^{\circ} \mathrm{C}$; the disordered phase (d-InGaP, high $E_{\mathrm{g}}$ ) at $725^{\circ} \mathrm{C}$ with a V/III ratio of 400 , both lattice matched to GaAs. The growth was performed using $\mathrm{PH}_{3}$, TMGa and "solution TMIn". For n-type doping $\mathrm{Si}_{2} \mathrm{H}_{6}$ was used. The structures were grown on (100) $6^{\circ}$ off towards (111) and (100) $2^{\circ}$ off towards (110) substrates.

The samples were electrically characterized by temperature dependent Hall-Van der Pauw measurements and $C-V$ measurements at $300 \mathrm{~K}$

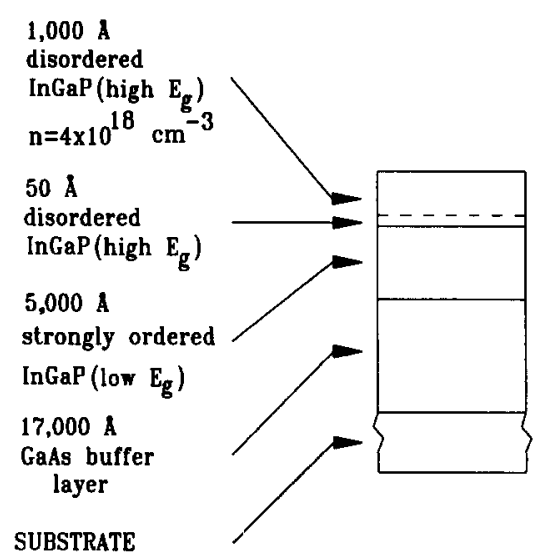

Fig. 1. Schematic drawing of the o-InGaP/d-InGaP homojunction.

using a Polaron plotter. The Shubnikov-De Haas $(\mathrm{SdH})$ measurements were performed in magnetic fields up to $20 \mathrm{~T}$ at $4.2 \mathrm{~K}$ in the High-Field Magnet Laboratory in Nijmegen.

The photoluminescence (PL) measurements were performed at $4.3 \mathrm{~K}$ with the sample in helium exchange gas. Optical excitation was provided by the $2.41 \mathrm{eV}$ line from an $\mathrm{Ar}^{+}$laser. The luminescence was dispersed by a $0.6 \mathrm{~m}$ double monochromater and detected by a cooled photomultiplier tube with a GaAs photocathode.

\section{Results and discussion}

The schematic structure of the samples is depicted in Fig. 1. It resembles conventional GaAs/ AlGaAs 2DEG designs in which the GaAs part is replaced by o-InGaP and the AlGaAs part by $\mathrm{d}-\mathrm{InGaP}$. The structure of the conduction band is depicted in Fig. 2, including the subbands in the potential well.

In Fig. 3 the $C-V$ profile, recorded in depletion mode, is shown together with the sample structure. Depletion of the 2DEG already starts at zero bias voltage, which is the case for 2DEG structures with no detectable parallel conductance [4]. The profile shows a high carrier density at the d-InGaP/o-InGaP interface, which decreases by one order of magnitude within $3 \mathrm{~nm}$ from the interface. The uncertainty in this mea- 

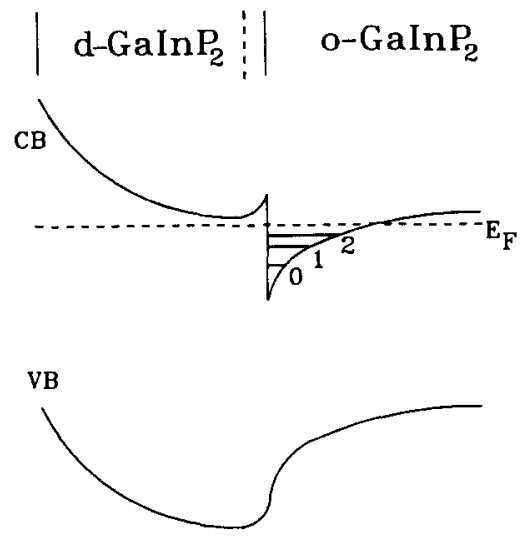

Fig. 2. Schematic drawing of the conduction band at the interface of the o-InGaP/d-InGaP homojunction; also drawn are three occupied subbands in the potential well.

surement is determined by the Debye length, which is about $5 \AA$ at these high carrier concentrations [5]. This measured value for the width of the 2DEG is an upper limit because of majority carrier diffusion; therefore strong spatial localization of the 2DEG is unambiguously shown here.

In Fig. 4 the $T$-dependence of sheet carrier density $\left(n_{2 \mathrm{D}}\right)$ and mobility $(\mu)$ is shown obtained from Hall measurements. The presence of the 2DEG at the o-InGaP/d-InGaP interface is confirmed by the absence of carrier freeze-out at

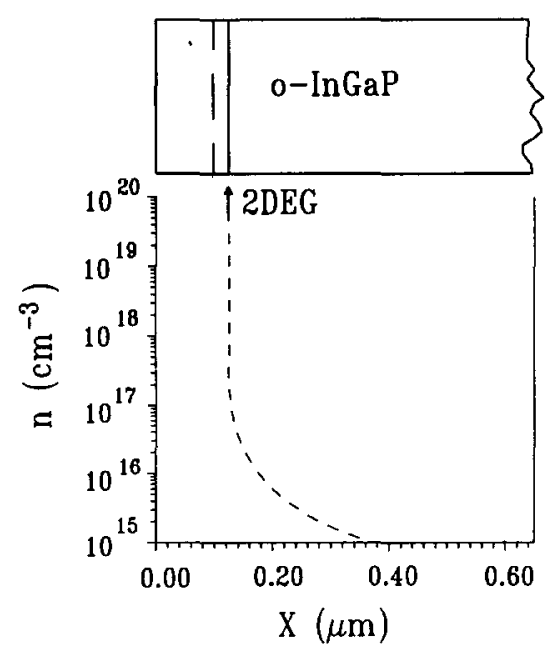

Fig. 3. $C-V$ depletion profile of the o-InGaP/d-InGaP homojunction. The position of the interface is sketched.

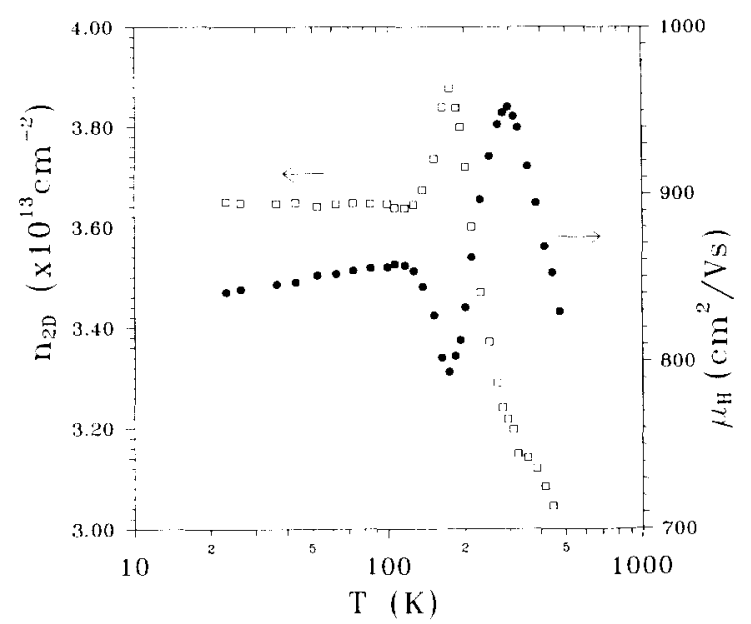

Fig. 4. Sheet carrier density $\left(n_{2 \mathrm{D}}\right)$ and mobility $(\mu)$ versus temperature (K).

$T<120 \mathrm{~K}$, i.e. the sheet carrier density is constant $n_{2 \mathrm{D}}=3.6 \times 10^{13} \mathrm{~cm}^{-2}$. When $T$ increases, $n_{2 \mathrm{D}}$ first increases as is previously observed in 2DEG systems [6]. At higher temperatures $(T \approx$ $180 \mathrm{~K}), n_{2 \mathrm{D}}$ starts to decrease slightly: at $T=300$ $\mathrm{K}, n_{2 \mathrm{D}}=3.2 \times 10^{13} \mathrm{~cm}^{-2}$. We believe that this abnormal decrease is typical for InGaP. At these temperatures carriers get delocalized from the ordered domains $[2,3]$ which will slightly broaden the conductive layer. However, the 2DEG is then still strongly confined, as was observed by the $C-V$ measurements. The value for $n_{2 \mathrm{D}}$ is extremely high, in fact it is the highest reported value for III/V structures, even higher than the values reported for $\delta$-doped structures which are known for their high $n_{2 D}$ [7]. The replacement of AlGaAs by InGaP is in our opinion the main reason for this. No DX centres are present in InGaP (when lattice-matched to GaAs), so no carrier reduction occurs due to trapping by them. At the interface, less non-radiative states are present due to the homoepitaxy. Finally, no aluminium is present in the structures, so no deep centres could be formed by the incorporation of reaction products of aluminium with oxygen and water. The high value for $n_{2 \mathrm{D}}$ of this homojunction implies that at least two subbands are occupied. 
No differences in electrical properties were observed between samples grown on the two different subtrate orientations, although samples grown on $(100) 6^{\circ}(1 \overline{1} 1)$ substrates possess only ordering in the [11ㅣ] direction, whereas layers grown on (100) $2^{\circ}(110)$ substrates show ordering in the two $\langle 111\rangle$ directions.

In Fig. 4, also the $T$-dependence of the mobility is plotted. The behaviour of $\mu$ versus $T$ contains various $2 \mathrm{D}$ features, as will be discussed in Ref. [8]. In summary, below $T=100 \mathrm{~K}$ the mobility is constant at a value of $850 \mathrm{~cm}^{2} \mathrm{~V}^{-1} \mathrm{~s}^{-1}$. Alloy scattering, even in the situation of several occupied subbands, cannot explain this low value. The behaviour of $\mu$ at $T<100 \mathrm{~K}$ can be explained by "mesoscopic" interface roughness scattering. At $T>100 \mathrm{~K}$, a fraction of the electrons gets delocalized from the ordered domains and scatter at them, thereby limiting the mobility. This cluster scattering mechanism has already been observed in InGaP [3] and explains the observed "U-shape" in Fig. 4 between 120 and $300 \mathrm{~K}$. At $T>300 \mathrm{~K}$, polar optical phonon scattering (two-dimensional) accounts for the observed behaviour.

The channel conductivity $\left(\sigma_{\mathrm{ch}}=e n_{2 \mathrm{D}} \mu\right)$ at room temperature is a parameter that requires maximization for high-speed-operation of electronic devices. In Table 1 , the $\sigma_{\mathrm{ch}}$ for this InGaP 2DEG is compared with that of 2DEGs in GaAs / AlGaAs, InGaAs/AlInAs, and $\delta$-FETs at $T=4.2$ and $300 \mathrm{~K}$. Despite its lower value at low $T$, the $\sigma_{\text {ch }}$ of $\mathrm{o} / \mathrm{d}$ InGaP heterojuction surpasses those of the others at $300 \mathrm{~K}$. This remarkably high value is caused by the $\mu(T)$ behaviour and makes the o/d InGaP 2DEG suited for application in

Table 1

Channel conductivities at 4 and $300 \mathrm{~K}$ for four different $2 \mathrm{D}$ structures

\begin{tabular}{lll}
\hline & $\sigma_{\mathrm{ch}}^{4 \mathrm{~K}}\left(\Omega^{-1}\right)$ & $\sigma_{\mathrm{ch}}^{300 \mathrm{~K}}\left(\Omega^{-1}\right)$ \\
\hline o/d InGaP $_{\text {GaAs } / A l G a A s}{ }^{\text {a }}$ & $5.0 \times 10^{-3}$ & $3.2 \times 10^{-3}$ \\
InGaAs $/ A l I n A s^{\text {b }}$ & $1.1 \times 10^{-1}$ & $1.1 \times 10^{-3}$ \\
$\delta$ FET $^{\mathrm{c}}$ & $9.4 \times 10^{-3}$ & $9.4 \times 10^{-4}$ \\
\hline
\end{tabular}

\footnotetext{
${ }^{a}$ From Ref. [9].

${ }^{b}$ From Ref. [10].

${ }^{\mathrm{c}}$ From Ref. [7].
}

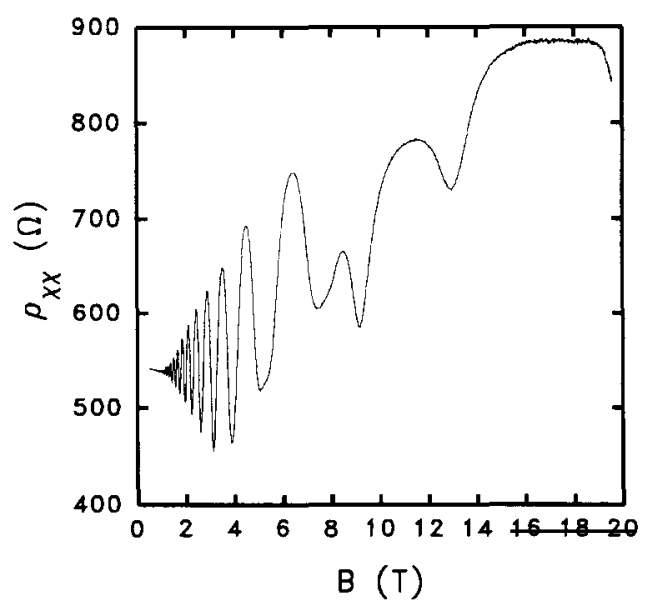

Fig. 5. Magnetoresistance $\left(\rho_{x x}\right)$ versus magnetic field (T) for the o-InGaP/d-InGaP homojunction.

electronic devices. A further increase of $\sigma_{\mathrm{ch}}$ can be expected if spacer thicknesses of $\sim 30 \mathrm{~nm}$ are used [9].

Shubnikov-De Haas measurements yielding the magneto-resistance $\left(\rho_{x x}\right)$ were performed at $4.2 \mathrm{~K}$ as a function of the magnetic field. As can be observed from Fig. 5, $\rho_{x x}$ starts to oscillate if $B>1 \mathrm{~T}$. At low $B$, the periodicity of the oscillations corresponds to $n_{2 \mathrm{D}}=7.6 \times 10^{11} \mathrm{~cm}^{-2}$. Above $4 \mathrm{~T}$, a second periodicity appears corresponding to a $n_{2 \mathrm{D}}=1.7 \times 10^{12} \mathrm{~cm}^{-2}$ (this is more pronounced when $\rho_{x x}$ is plotted versus reciprocal $B)$. The $n_{2 \mathrm{D}}$ values obtained from these measurements are much lower than the value obtained with Hall measurements $\left(n_{2 \mathrm{D}}=3.6 \times 10^{13} \mathrm{~cm}^{-2}\right)$. This points at the occupancy of at least three subbands; the observed periodicities in the SdH measurements are due to the higher subbands. This view is supported by the behaviour of $\rho_{x y}$ (Hall configuration) at low fields that yields the total sheet carrier density [8]. Stronger magnetic fields are necessary to reveal oscillations in $\rho_{x x}$ of the lowest subband, because the width of its Landau levels is larger than the splitting between them at magnetic fields of $20 \mathrm{~T}$. Measurements at higher fields will be performed in the near future.

In Fig. 6, the $4.3 \mathrm{~K}$ PL spectrum of the structure grown on the (100) $6^{\circ}(1 \overline{1} 1)$ substrate is shown. The peak of the d-InGaP is seen at the 


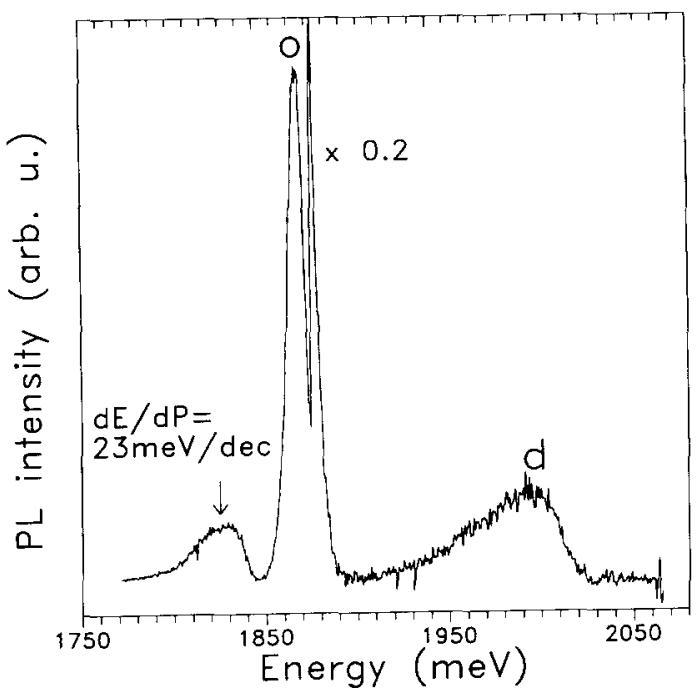

Fig. 6. Photoluminescence spectrum recorded at $T=4.3 \mathrm{~K}$; indicated are the peaks involving d-InGaP (d), o-InGaP (o) and the 2DEG (arrow).

high energy side of the spectrum. About $120 \mathrm{meV}$ lower in energy, the peak of the o-InGaP is observed, exhibiting characteristic properties such as the inverted S-shape [2]. At $\approx 1.82 \mathrm{eV}$, a small peak is observed, corresponding to emission from $2 \mathrm{D}$ electrons with spatially separated holes. With increasing power density, this peak shifts towards higher energies with a $\mathrm{d} E / \mathrm{d} P$ of $23 \mathrm{meV}$ per order of magnitude of excitation density because of the increasing overlap between the wavefunctions of electrons and holes. A detailed optical study of these samples will also be presented elsewhere [8].

\section{Conclusions}

We have grown for the first time modulation doped o-InGaP/d-InGaP homojunctions and demonstrated the presence of a 2DEG at the interface. The outstanding technique for growing these structures is MOVPE. The structures show extremely high sheet carrier densities and con- stant mobilities $\left(\mu \approx 850 \mathrm{~cm}^{2} \mathrm{~V}^{-1} \mathrm{~s}^{-1}\right)$ if $T<100$ $\mathrm{K}$. $C-V$ measurements at room temperature showed that the dense $2 \mathrm{DEG}$ is strongly confined at the $\mathrm{o} / \mathrm{d}$ interface. The $T$-dependence of the mobility is determined by three scattering mechanisms. At $T<100 \mathrm{~K}$, interface roughness scattering limits $\mu$ and in the range $100<T<300 \mathrm{~K}$, cluster scattering at the ordered domains is the important scattering mechanism. At $T>300 \mathrm{~K}$, polar optical phonon scattering determines $\mu$.

Shubnikov-De Haas measurements showed oscillations of $\rho_{x x}$ in reciprocal field; two excited subbands were observed. The low field $\rho_{x y}$ data yielded the total $n_{2 \mathrm{D}}$ again. The 2DEG was also observed in PL measurements.

\section{Acknowledgments}

E. Dijk and P. van Rijsingen are gratefully acknowledged for processing the samples. This work was financed by NOVEM, Project No. 146.100-041.1 and by FOM.

\section{References}

[1] S.-H. Wei and A. Zunger, Appl. Phys. Lett. 56 (1990) 662.

[2] F.A.J.M. Driessen, G.J. Bauhuis, S.M. Olsthoorn and L.J. Giling, Phys. Rev. B 48 (1993) 7889.

[3] G.J. Bauhuis, F.A.J.M. Driessen and L.J. Giling, Phys. Rev. B 48 (1993) 17239.

[4] E.F. Schubert, K. Ploog, H. Dämbkes and K. Heime, Appl. Phys. A 33 (1984) 63.

[5] P. Blood, Semicond. Sci. Technol. 1 (1986) 7.

[6] R. Dingle, H. Störmer, A. Gossard and W. Wiegmann, Appl. Phys. Lett. 33 (1978) 665.

[7] N. Pan, J. Carter, G.S. Jackson, H. Hendriks, J.C. Huang and X.L. Zheng, in: GaAs and Related Compounds 1990, Inst. Phys. Conf. Ser. 112, Ed. K.E. Singer (Inst. Phys., Bristol, 1991) p. 401.

[8] F.A.J.M. Driessen, G.J. Bauhuis, P.R. Hageman and L.J. Giling, to be published.

[9] W. Walukiewicz, H.E. Ruda, J. Lagowski and H.C. Gatos, Phys. Rev. B 30 (1984) 4571.

[10] A. Kastalsky, R. Dingle, K.Y. Cheng and A.Y. Cho, Appl. Phys. Lett. 41 (1982) 274. 